\title{
Mine-Scale 3D Stress Model for the New Mine Level Project, El Teniente Mine, Codelco, Chile
}

\author{
J. Jarufe Codelco, Chile \\ P. Vasquez Codelco, Chile
}

\begin{abstract}
For the development of the pre-feasibility study for the New Mine Level Project (NML) carried out by Codelco Chile Project Corporate Vice-Presidency, it is fundamental to have numerical models that allow estimating the stress state for the various mining options under study in a quick, simple and reliable manner. For this reason, a simplified mine scale model was created to analyse geomechanical issues that go from drift scale (overbreaking), large excavations (caverns) to mine scale issues (caving front geometries, mining macro-sequences, sector interactions). The software tool preferred was Map3D (Mine Modelling Pty Ltd).
\end{abstract}

The boundary conditions used as the base for the Map3D modelling tool correspond to the geometry to be analysed and the pre-mining stress state in the zone of interest. To estimate this latter condition, the Conceptual Stress Model (Karzulovic et al., 2006) information was used and originally, the stress orientation expected for that zone based on Plate Tectonics (east-west trending) was considered. To test the model's efficacy, various validation exercises were carried out: comparison of the model's results with field stress measurement for different years, between the abutment stress zone predicted by the model and the one observed in the field, and the model-predicted overbreak versus the actual overbreak obtained through field surveys.

It can be concluded that the model created fulfils the reliability levels that have been historically used for mine-scale models in a pre-feasibility engineering stage. The validated and calibrated boundary conditions can be applied both for mine-scale and drift-scale models, with error values of $40 \%$ compared to stress measurements and $20 \%$ when compared to field-observed damage.

\section{Introduction}

The New Mine Level Project involves the extraction of reserves below the current El Teniente. This new deeper mine will be affected by higher stresses than the current mine and the damage zones are also expected to be greater. For the estimation of the stress magnitudes at the New Mine Level Project depths, two different mine-scale models have previously been developed, a conceptual stress model (Karzulovic et al., 2006) and a 3DEC rough mine-scale model (Itasca, 2007). However, the lack of detail of these models makes them unsuitable for studying detailed damage extension and geometry based issues. Therefore, a simplified minescale model was created to quickly and reliably analyse geomechanical issues that go from drift scale (overbreaking), large excavations (caverns) to mine scale issues (caving front geometries, mining macrosequences, sector interactions).

\section{Mine-scale models for the New Mine Level}

The construction of a mine-scale model is a complex task, as a single model must have the capacity to represent both the mine-global behaviour and the influence of excavations at drift scale. It is particularly difficult to achieve this result because it is almost impossible to simulate all the heterogeneities and discontinuities of the rock mass, so that the model can be calibrated to the information available, such as stress measurements, drift overbreak, damage ahead of the caving fronts, etc. This variability in the rock mass makes it very difficult to build a model of such a large scale with such small comparison parameters (mainly stress measurements) used for validation. 
As a first approach to the definition of the stress field behaviour in the New Mine Level, the El Teniente Mine Conceptual Stress Model was developed (Karzulovic et al., 2006). This generated a series of charts that allow estimation of the magnitude of the stress state in the mine for different elevations using existing stress measurements and simple 2D numerical models. In addition to this, a graphical representation of the stress trajectories in the mine was created, which allows an estimate of the principal stresses orientation and inclination. One of the results of this conceptual model is shown in Figure 1.

Although the conceptual model gives an estimate of the pre-mining stress state regarding magnitude and orientation, it became necessary to have a more complete model that is capable of simulating, with a known confidence level, the stress redistribution resulting from the mining activity. This model was necessary in order to evaluate different mining macro sequences from the viewpoint of damaged zones, induced stresses, interaction between sectors, face geometries and other factors. To satisfy this need, a second model, the New Mine Level Stress 3D and Mining Macro Sequences Model was developed by Itasca (2007). This mine-scale distinct elements model generated by the 3DEC software includes the different mine lithologies and the most important structural systems. It also included mining activities from 1990-2005, in five-year intervals, in order to calibrate and validate the results with stress measurements made at El Teniente mine. The results of the calibration and validation process gave a mean error when comparing with stress measurements of $37 \%$ (Equation 2). Calibration and validation based on observed damage was not very practical due to the large size of the elements in the model (minimum $40 \mathrm{~m}$ ). The resolution of the model was too coarse to be able to see detail of damage.

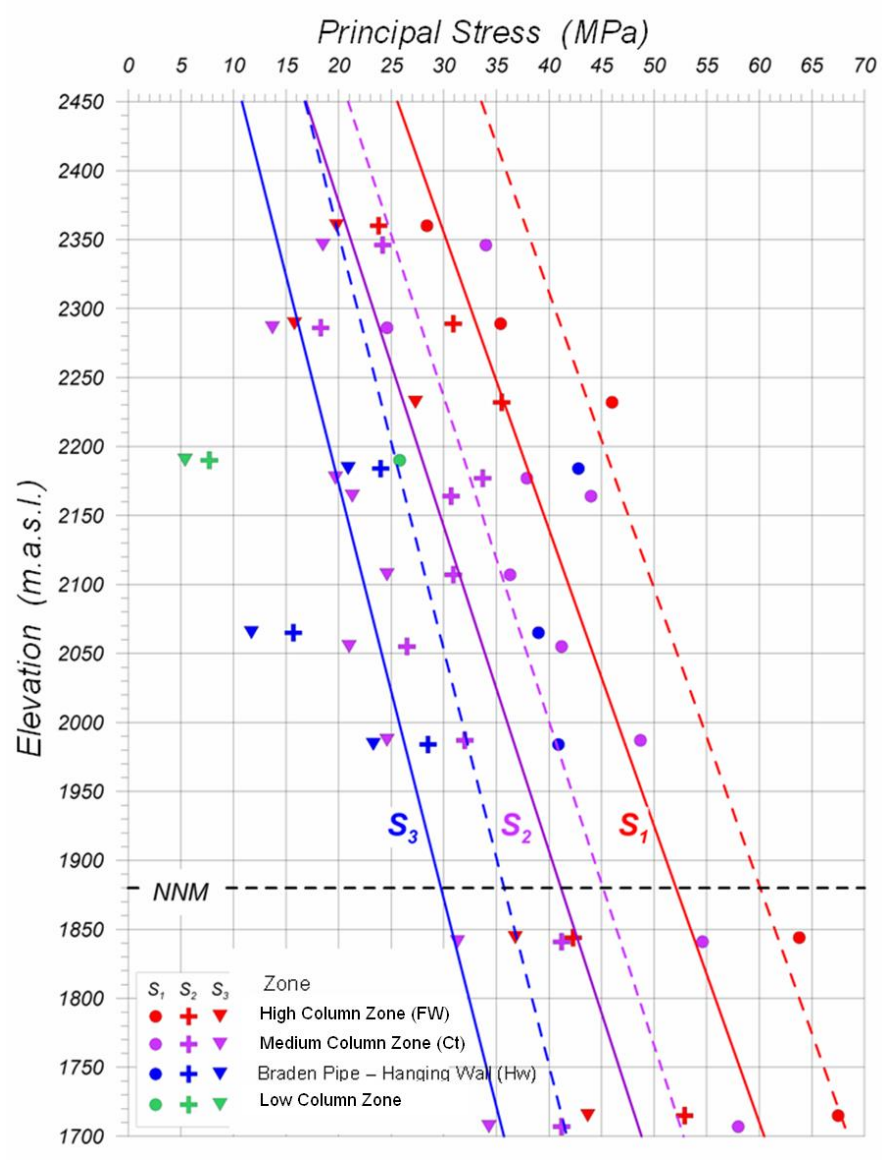

Figure 1 Stress distribution for the virgin stress state (points indicate stress measurements, solid lines correspond to best fit lines for the medium column zone and filled lines, to the high column zone) according to the conceptual stress model (Karzulovic et al., 2006) 


\section{Development of the mine-scale Map3D model}

The third model constructed was the mine scale Map3D model. For modelling purposes, the rock mass was considered as an elastic, continuous and isotropic medium, including only two lithologies. CMET, the predominant lithology, was modelled as an infinite medium and the Braden Breccia (an intrusive body that is unmined) was defined by means of fictitious force elements. In addition to this, a surface excavation was generated to include the effect of topography in the model (Figure 2). The broken material generated by caving was considered as void in the model, so the craters generated by subsidence correspond to excavations that reach the surface. Omitting the broken material improves the model's simplicity; however this means that the results obtained increase the error between the values of the in situ measured stresses and the ones resulting from the model. This must be taken into account when analysing and interpreting such results.

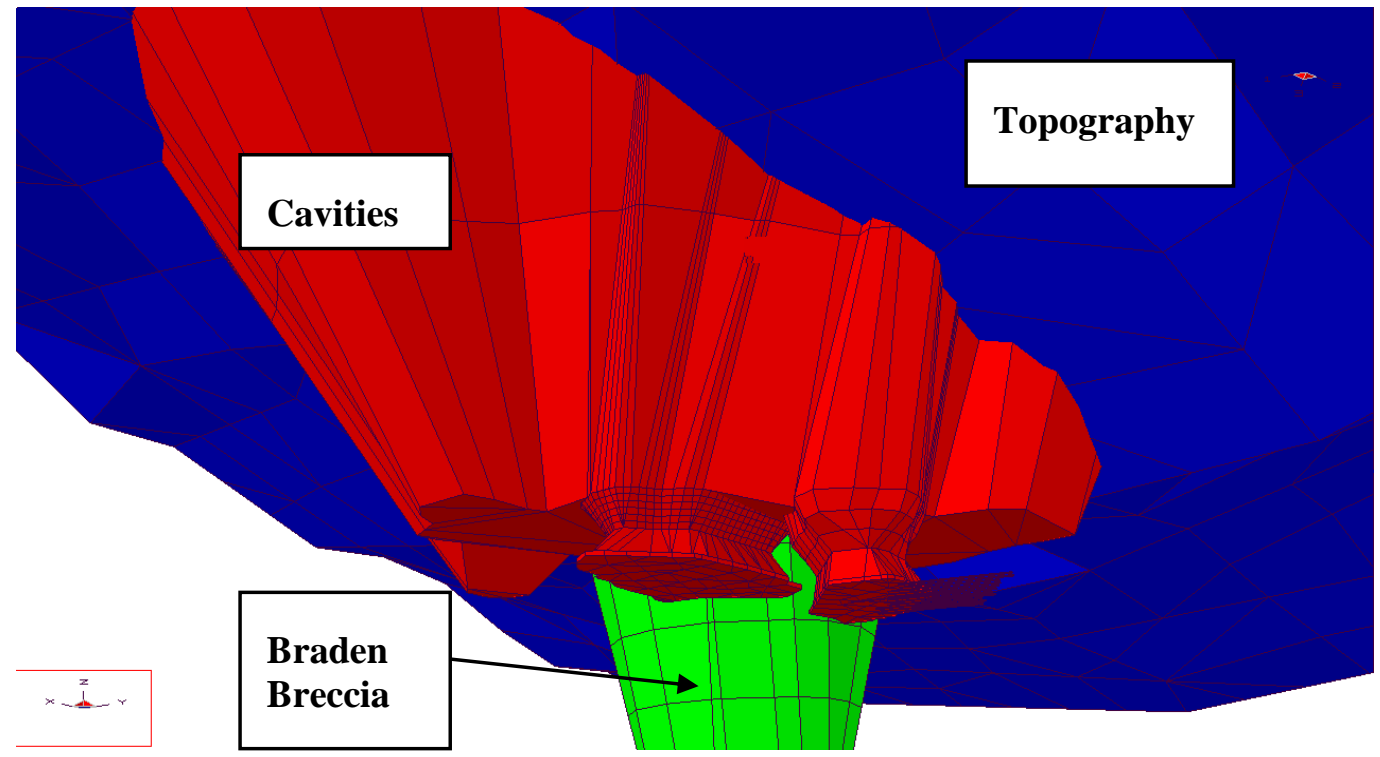

Figure 2 Map3D model with surface topography, Braden Breccia and cavities (2005); view from below, from NE-SW

\subsection{Constitutive model}

For the development of the mine-scale model presented here, an elastic constitutive model was used. This only solves the equilibrium, continuity and elasticity equations, which are expressed in terms of the stresses $\sigma_{\mathrm{ij}}$ and strain $\varepsilon_{\mathrm{ij}}$ (Wiles, 2007). In this manner, the model's input parameters to calculate the stress-strain field are the initial stress condition (pre-mining), Young's modulus E and Poisson's ratio $\mu$, so the calibration and validation process is relatively simple and quick compared to other constitutive models (plastic, strainsoftening and others) that also consider rock strength properties. The main advantage is that elastic models do not consider the rock mass strength properties and, only in case there are multiple materials, elastic modulus plays an important role. Because the only variable is the pre-mining stress state (besides the geometry defined by the problem), the input data is simplified and reduces the total error of the result. In this manner, the total expected error depends on the error of the considered geometry (controllable and known factor), of the stress field (known factor) and of the numerical model (controllable factor).

The main disadvantage of the elastic model is the numerical impossibility to simulate the stress redistribution once the rock strength is exceeded. This is particularly important for the pillar and drift overbreak study. The important issue is to define how significant these inelastic effects are on the results. This can be carried out during the calibration stage and, if it is determined that errors due to these effects are important, plastic modelling should be considered in the analysis. For the prefeasibility level of engineering, the inelastic effects do not cause significant errors in the results, so the elastic modelling option is considered as adequate. 


\subsection{Geometries considered}

The model created considers different geometries depending on the year under study and the scale of the problem to analyse. In this manner, in order to compare the model's results with the results of the stress measurements, global models for the entire mine were prepared for years 1990, 1995 and 2000. These global models were prepared based on the break area defined by all drawpoints with an extracted percentage higher than 30\% (shaded area in Figure 3(a)) and on the respective subsidence angles. For years 1999, 2005 and 2007 models up to caving front scale were prepared, including the caving front in detail and in some cases including the undercutting or exploration drifts. The detail models only correspond to the specific sectors located to the South and East of the Braden Breccia. They correspond to Diablo Regimiento in 2007, Ten-4 Sur, Esmeralda and Reservas Norte sectors 2001, 2005, 2007. In addition, nearby sectors that could influence the stress state of the zone such as Ten-5 Pilares and Isla LHD were included for these models. These detailed models were built based on damage drawings with information about the location and shapes of the extraction fronts, undercutting and 30\% draw (Figures 3(b) and (d)).
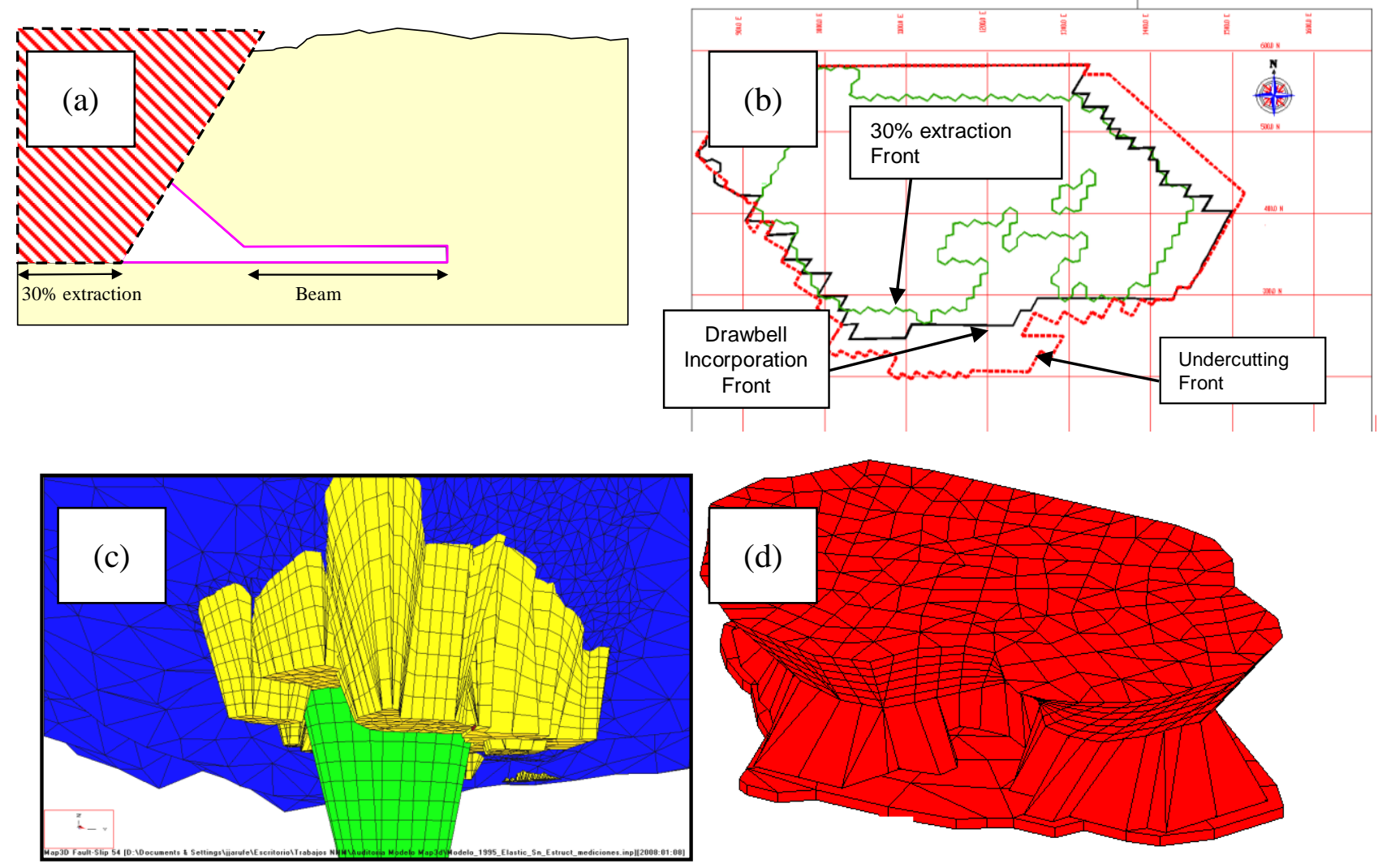

Figure 3 Conceptual representation of the $30 \%$ extraction based models (a) and (c) and the mine geometry data for the detailed models (b) and (d), including undercutting front, $30 \%$ extraction front and drawbell incorporation front

As a general rule, drifts and infrastructure works were not included in the detailed models, because the main objective was to study the effect of the caving front in the stress distribution and not the specific effect on the drifts at different levels. However, some drifts were included in the undercutting level at Reservas Norte sector and the exploration drift at the Ten- 8 haulage level in order to validate the mine model.

\subsection{Geology and properties considered}

For the development of the mine-scale model, only the lithological units that most influence the behaviour of the stress field were considered. In this manner, the modelled lithologies were the CMET $(80 \%$ of the deposit) as an infinite medium and the Braden Breccia as a fictitious forces volume. The effect of topography was considered by modelling a void volume in the upper part of the model. The geotechnical properties used 
correspond to the ones defined in the Geology and Resources for the New Mine Level report (Celhay et al., 2005) and are shown in Table 1.

Table 1 Rock mass elastic properties used in the Map3D model

\begin{tabular}{ccc}
\hline Elastic Parameters & CMET & Braden Breccia \\
\hline $\mathrm{E}(\mathrm{GPa})$ & 42 & 21 \\
$\mu$ & 0.19 & 0.24 \\
\hline
\end{tabular}

As the model is developed under elastic constitutive relationships, the only properties that influence the stress distribution correspond to the Young's Modulus E and Poisson's ratio $\mu$, so these are the only input parameters for Map3D. The rock strength parameters are only used once the model has been solved to make comparisons between strength and load (for example, safety factor based on a failure criterion).

\subsection{Stress state in initial condition (pre-mining)}

The stress state in Map3D is calculated based on the boundary conditions imposed by the user (Wiles, 2006). These conditions correspond to the different surfaces defined by the boundary elements (excavations) and by the pre-mining stress field in order to satisfy:

$$
\sigma_{\text {Excavation boundary }}=\sigma_{\text {Pre-mining }}+\sigma \mathrm{BEM}
$$

In this equation, the objective is to make the radial stresses at the boundaries of the excavations equal to zero. This is achieved by modifying the pre-mining stress state according to the continuity, elasticity and equilibrium Equations that are solved through the boundary element method (BEM).

The pre-mining stress state must be established before introducing any mining activity in the zone. To evaluate this pre-mining stress, it is not enough to eliminate the stress measurements that are near the caving fronts, because there is always an influence from other sectors of the mine (especially in the orientation of the stress measurement); so the stress measurements are not a good indicator of a pre-mining stress state.

The required stress state is defined by the gradient of the principal stresses as a function of elevation and the orientation of these stresses in terms of the trend (azimuth) and plunge (dip) of a line. For the calculation of the stress gradient, the results submitted from the El Teniente Mine Conceptual Stress Model (Karzulovic et al., 2006) were used. From the curves obtained from this model (Figure 1), it is possible to obtain an Equation that defines the variation of principal stresses at deeper levels. This corresponds with the first input for the initial stress field condition.

To establish the orientation of the principal stresses, central Chile plate tectonic condition was considered as the principal stress orientation, so according to Figure 4 the major principal stress orientation is east-west and trend and plunges west at $15^{\circ}$. The intermediate principal stress has a north-south sub-horizontal orientation and the minor principal stress has a sub-vertical orientation, equating in magnitude to the lithostatic weight of the rock mass.

It is important to note that the stress gradient used as input for Map3D was achieved as a product of an iteration process between the best fitting Equation that adjusts the stress magnitude gradient in depth and the Map3D results. This is because the stress gradient obtained from the best fit curve is not exactly achieved in the numerical model and the best fit curve must be adjusted, based on the numerical modelling results. This leads to a second 'best fit' curve which is again used as Map3D input data. This recursive process leads to an optimisation of the stress gradient obtained from the numerical simulation, obtaining the best results for the pre-mining stress gradient.

Once the initial stress field is fixed, mine geometries can be included in the model. In this way, the stress field matches up with the different cavities (associated with different dates) existing during the analysis of the problem. 


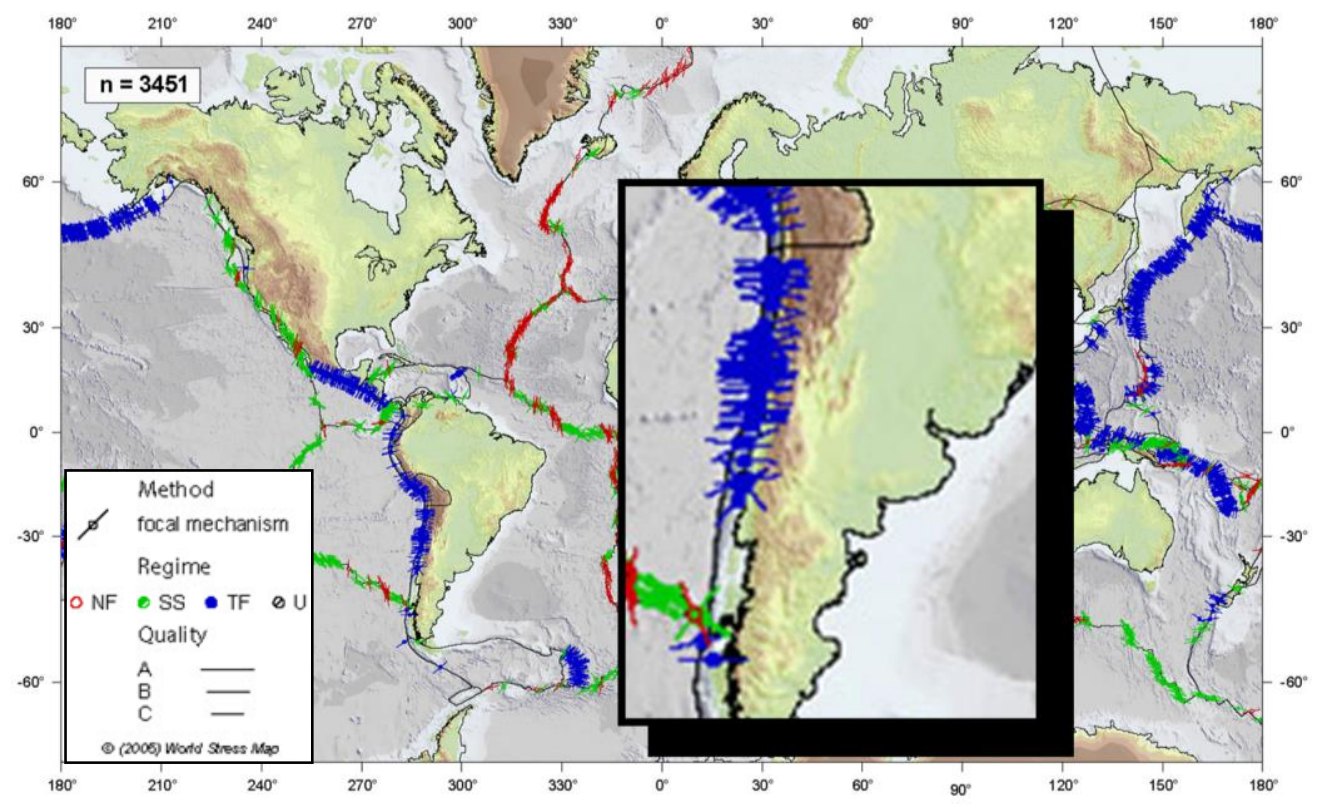

Figure 4 Stress orientations according to the World Stress Map, indicating an east-west principal stress orientation for the central Chile sector

\subsection{Model discretisation}

The different surfaces that define the problem must be discretised into finite surfaces, which can be defined numerically and thus be included in the different equations solved by the BEM.

The size of the elements formed establishes the detail or resolution of the results. Considering this, the Map3D discretisation varies in space, concentrating more elements in the zones that need more detail (such as drifts, caves or other infrastructure) and a lower density of elements for the global stress state estimates (mine-scale stress estimates). Together with the mine geometry, the results grid at which the results will be calculated must also be discretised. As in the case of geometry, a higher element density generates higher detail in the results. The generated Map3D model considers a mine geometry discretisation that goes from $20 \mathrm{~m}$ elements in zones far from the levels under study up to $1.5 \mathrm{~m}$ elements in the zones that define drifts and other infrastructure. For the results grid layout, an element size of 4-10 m was considered for global stress state estimates and element sizes of $0.1-3 \mathrm{~m}$ for the study of more detailed works (Figure 5).

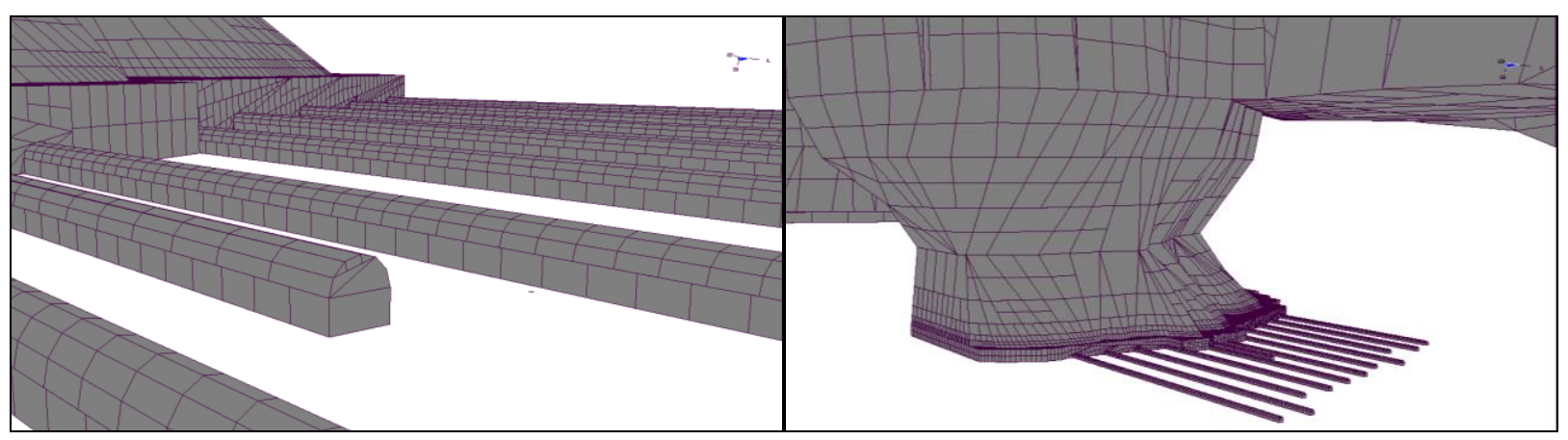

Figure 5 Variable discretisation has been used to study both mine and drift scale problems

\section{Validation and reliability of the Map3D model results}

Once the model is generated and executed, the reliability of the results obtained must be established in order to make future predictions with a known margin of error. This is achieved through different model validation exercises, by solving problems with a known response in order to evaluate the differences and to take them into account in predictive exercises. Basically, two types of exercises were carried out. They were the 
comparison of the stress measurement results obtained in the mine in different years with the model's results and the comparison between the field-surveyed information (abutment zone and drift overbreak) and the information provided by the model. The error obtained from these comparisons with existing cases was assumed to also apply to a margin of error to be established for the results obtained, assuming that the error range will be maintained for predictive models.

\subsection{Comparison with stress measurements in the mine}

For the validation based on the mine stress measurements, complete mine models were used for 1990, 1995 and 2000. Only a single more detailed local model was used for 2005. The methodology was as follows:

\subsubsection{Selection of stress measurements for validation}

The first step consists of obtaining a database of the stress measurement results not affected by the geometry of the mining front (because the model is built based only on $30 \%$ extraction) and with a date compatible with the year of the model under study. This means that from the viewpoint of time, the measurements made in the year analysed will be considered in addition to the non-induced measurements made before the date. In other words, a measurement of 2005 that is not affected by mining that year is not affected by mining in 2000 or other former years. Also, measurements made near mining fronts and located in lithologies in which elastic modulus differs by more than $30 \%$ of the value of this parameter in the CMET were eliminated. This is because the stresses induced by the caving front (which geometry was not modelled in detail) affect these bodies in a different manner and this was not represented in the model. For 2005, only the measurements made during that year were considered because that year the geometry was modelled in higher detail. This is summarised in Table 2.

Table 2 Results obtained from using the Itasca (2007) formula to compare actual and modelled measurements

\begin{tabular}{ccc}
\hline Year & Stress Measurements Considerated & Stress Tensor Error \\
\hline Pre-Mining & 39 & $40 \%$ \\
1990 & 89 & $43 \%$ \\
1995 & 59 & $38 \%$ \\
2000 & 32 & $42 \%$ \\
2005 & 10 & $26 \%$ \\
\hline
\end{tabular}

\subsubsection{Selection of the comparison technique}

To compare the actual data with that calculated by the model, the relationship used by Itasca (2007) was used, which is defined by:

$$
\varepsilon=\sqrt{\frac{\sum\left(\Delta \sigma_{\text {model-measured }}\right)^{2}}{\sum\left(\sigma_{\text {measured }}\right)^{2}}}
$$

This equation is an estimation of the deviation of the modelled data compared to the actual data (error). This error, when calculated for known situations, defines the model's reliability in terms of the range of the actual result compared to the modelled value. Using this approach, the total error obtained for the different models can be seen on Table 2 .

The results obtained are quite satisfactory considering the low complexity of the model used. If, in addition, it is considered that the natural rock variability in stress measurements is about \pm 20 to $\pm 30 \%$ where, particularly for El Teniente, measurements from a same drillhole have a variation of up to 58\% (Itasca, 1998) with a mean variation of $23 \%$, it can be considered that the model error level obtained is adequate for a prefeasibility engineering. As a comparison, the model with different lithologies, structures and using an elastoplastic constitutive model developed by Itasca (2007) obtained a mean error level of $37 \%$. 


\subsection{Comparison with field information}

Although comparison with stress measurements allows verification of the model error, it is also important to know how the model can simulate the damage zones observed in the mine, whether in form of abutment or overbreak. In this manner, a comparison of the damage zones estimated by the model and the ones actually observed was made.

\subsubsection{Abutment zones estimation}

To determine the field damage from the mine-scale model, it first became necessary to define a damage criterion that allows differentiating the intact sectors from the ones that have damage. To do this, the stress state at a certain point in the model is compared with the Hoek and Brown strength envelope (Hoek et al., 2006), identifying the sectors where the over-stress condition occur (failure of the rock mass for stresses above the envelope). The over-stress index calculation is made based on the pair of values of $\sigma_{1}$ and $\sigma_{3}$ obtained from the mine scale model. These values are compared to the $\sigma_{1}$ and $\sigma_{3}$ pair of the failure envelope, evaluating in this manner the overstress at each point of interest, as shown in Figure 6.

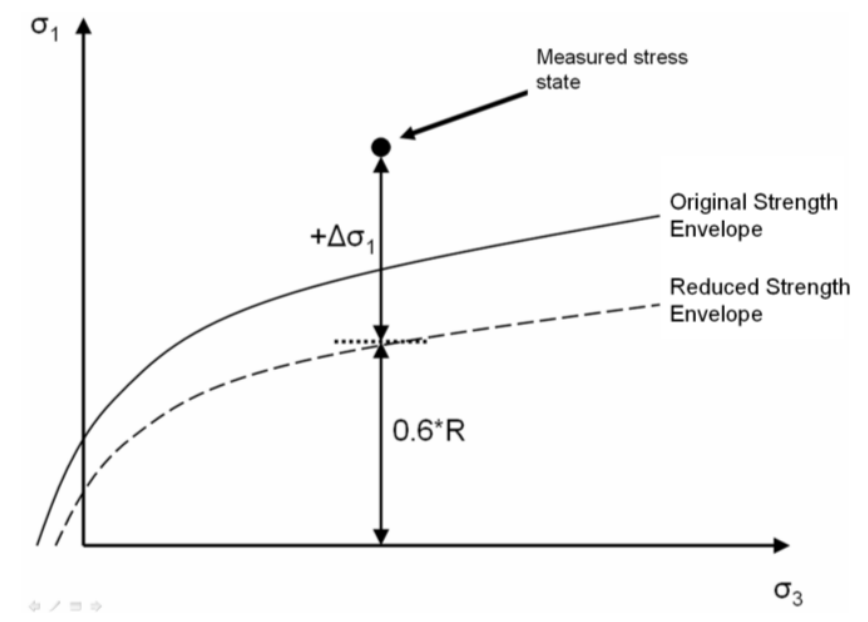

Figure 6 The reduced strength envelope used according to the over-stress criterion defines a larger over-stress area ahead of the caving front. This is due to a reduction of about $40 \%$ in the rock mass strength properties and such a penalty to the properties is due to the effect of the caving front on the rock mass quality near the mining sectors

This comparison led to the conclusion that the most representative over-stress index for strong damage observed in the field is the value of 1.7 , meaning that in damage areas the rock mass strength has a penalty of about $40 \%$ (keeping $60 \%$ of its original value). This penalty value on the rock mass strength properties is empirically determined and it can be explained as the deterioration suffered by the rock mass due to the effect of the caving front move ahead, which in our case is translated into a reduction of the Geological Strength Index, GSI (Vasquez et al., 2008b) when using the Hoek and Brown failure criterion (Hoek et al., 2006). Figure 6 shows a schematic representation of the over-stress failure criterion.

The calibration/validation results for the Esmeralda sector in 1999 show that the over-stress criterion represents the damage observed in the $\mathrm{Fw}$ zone of that sector. Also, in sectors Ten Sub-6/RENO and Esmeralda, modelled for 2007, the criterion represents the damage caused by the stress redistribution (abutment stress), mainly due to the compression applied on the rock mass caused by the high $\sigma_{1}$ and $\sigma_{3}$ values (Figure 7). 


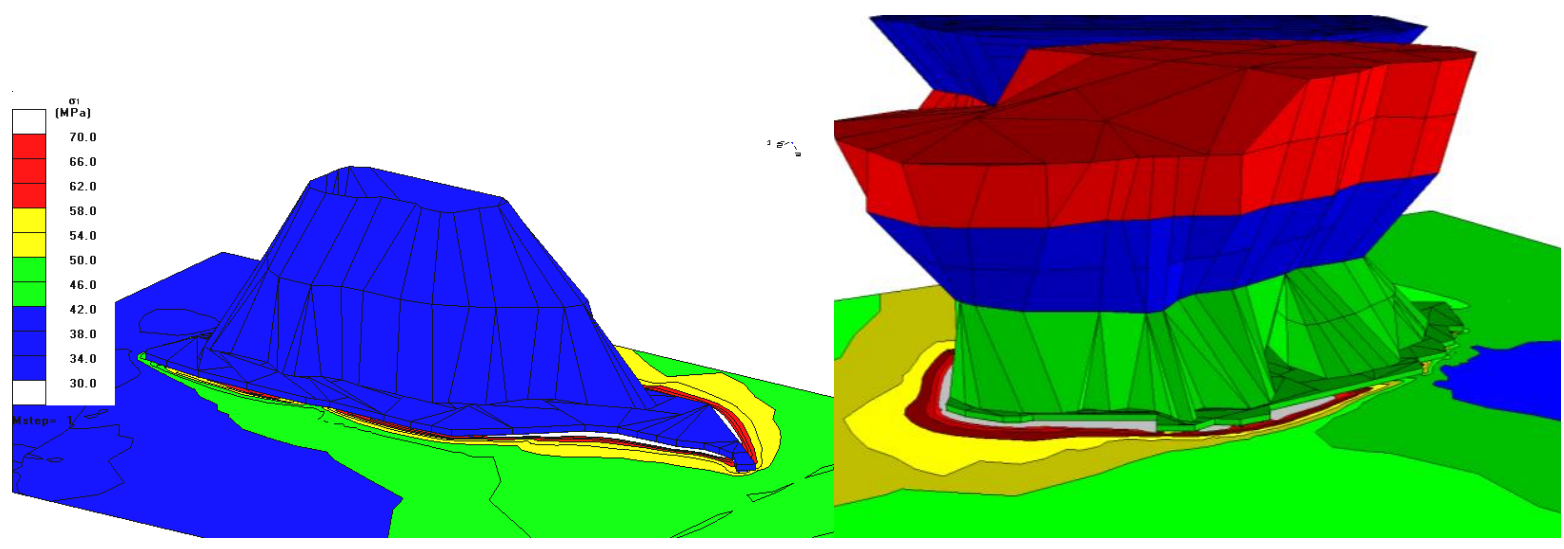

Figure 7 Major principal stress $\left(\sigma_{1}\right)$ redistribution for the some geometries studied as back analysis based on the mine scale model developed for the NML in Map3D

The models consider the entire geometry of the sectors studied, except for the drifts and existing infrastructure (for example, ore passes or others). This creates some differences between what has been modelled and the field observations. However, these differences are minor and the damage provided by the model can be considered as a good and reliable representation of what is observed in the field (Figure 8).

\subsubsection{Drift overbreak}

As a validation exercise for a mining front scale, the field-observed overbreak was compared with the Map3D model-estimated overbreak (Vasquez et al., 2008a). For this purpose, a safety factor was used to determine a cut-off value considering actual data. The drifts considered in the exercise corresponded to the XC-60 Fw exploration drift located in Ten-8 level and the undercutting level drifts in Reservas Norte mine.

When studying the overbreak in the Ten-8 exploration drift, it was observed that the safety factor that best represents overbreak corresponds to the value of 1.3. This value best matches the shape and extent of the damaged zone. While actually there is an overbreak of $0.6 \mathrm{~m}$ in the upper West wall, the model shows an overbreak of $0.65 \mathrm{~m}$ in that same location. The safety factor of 1.3 obtained from the exploration drift study in Ten-8 was also applied to the Ten Sub-6/Reservas Norte modelled drifts - with the rock strength properties found in the sector under study - and the modelled results agree quite well with field evidence (Figure 9). The evidence that the extent of the modelled overbreak is near the actual one corresponds to the fact that the magnitude of the modelled stresses is near the existing stresses in the sector under study. 
(a)

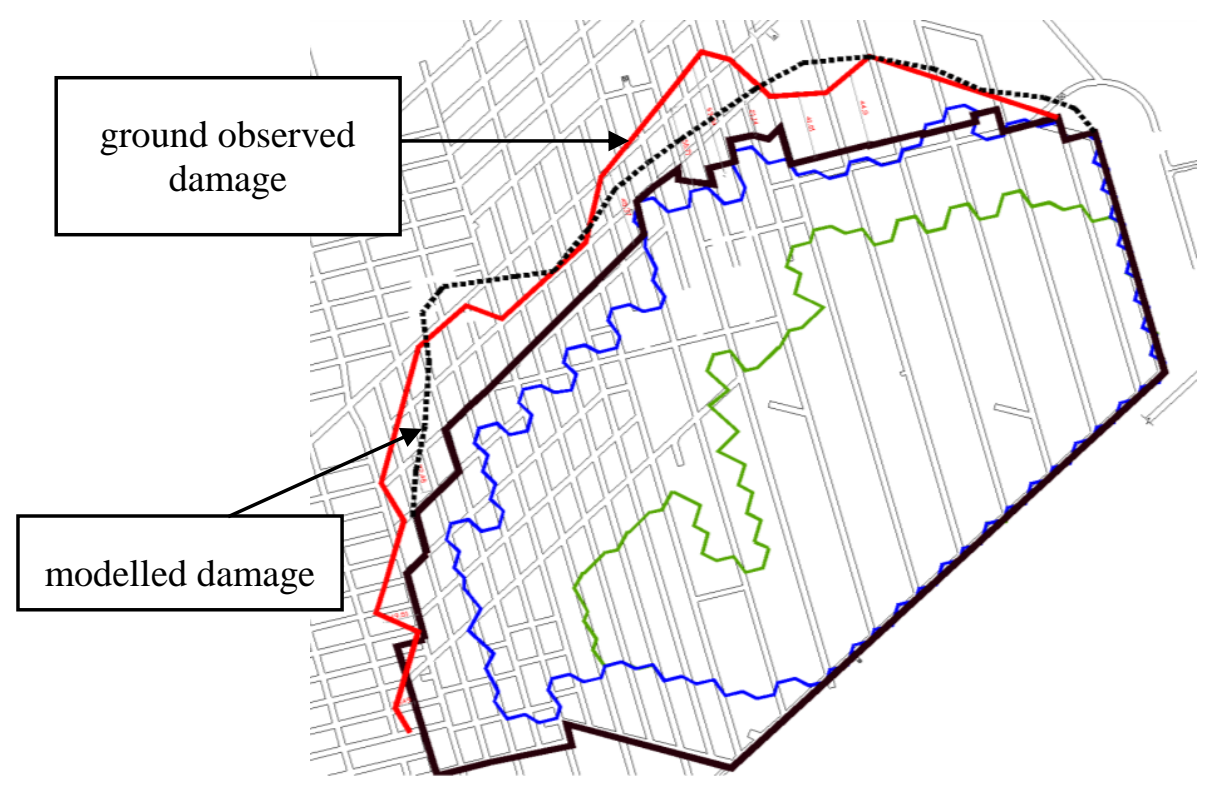

(b)

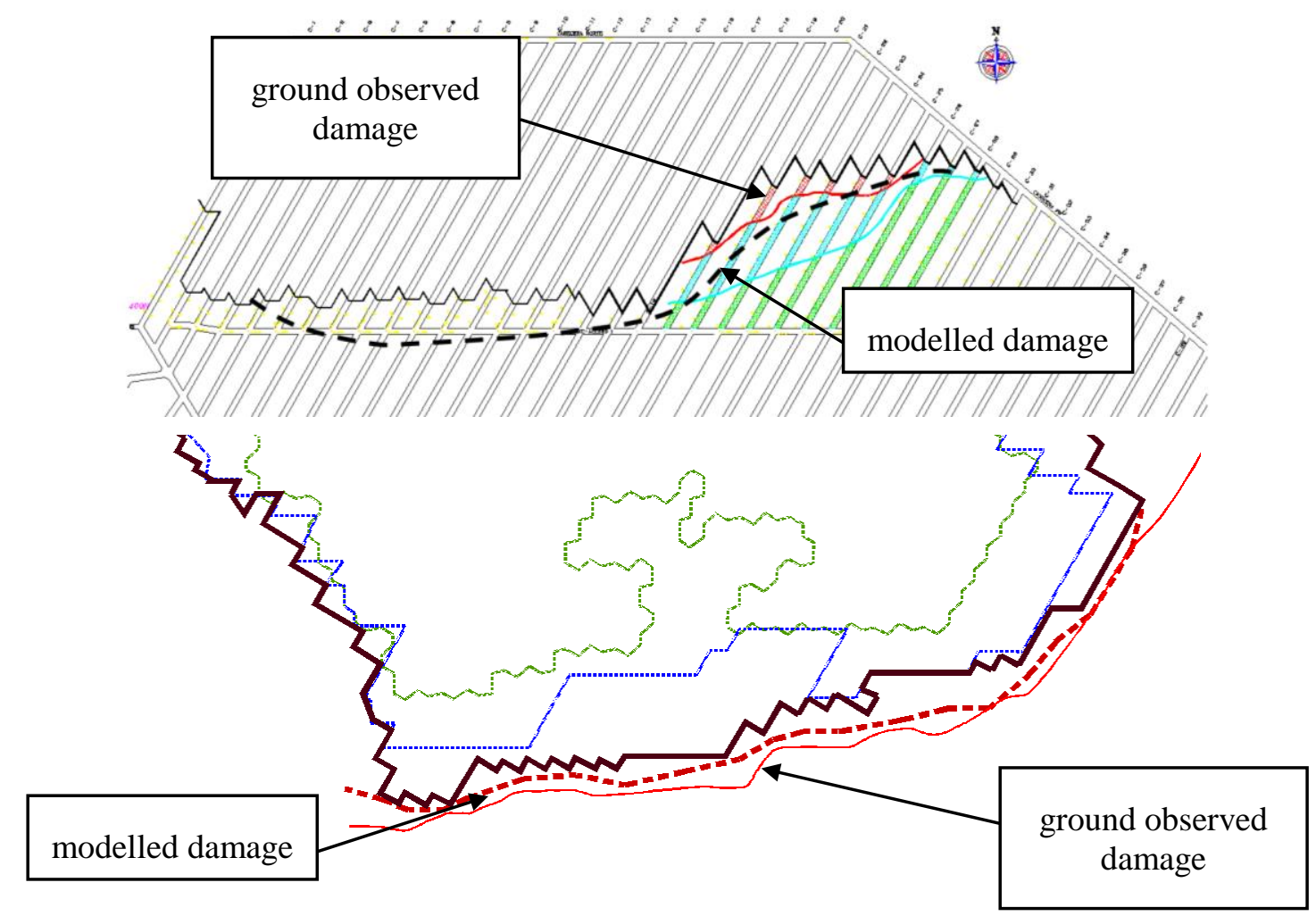

(c)

Figure 8 Comparison between the results obtained by means of the over-stress criterion for a value equal to 1.7 (segmented line) and the strong damage reported by the SGM-GRMD Operational Geomechanics Area (solid line) for sectors (a) Ten Sub-6/RENO 2007; (b) Esmeralda 1999; (c) Esmeralda 2007. The coarse solid line corresponds to the undercutting front

On the other hand, the location of the overbreak zone is mainly related to the stress orientation in the sector (overbreak mainly results from the stress state, not from the structural systems), whether they are pre-mining or induced stresses. For the prediction of the future behaviour of the rock mass in terms of damage visible in the field as an overbreak or abutment stress damage, the estimated error is about $20 \%$ considering Equation (2) which allows for making good estimates of the likely rock mass failure due to the mining geometry (front shape and extent, mainly). 


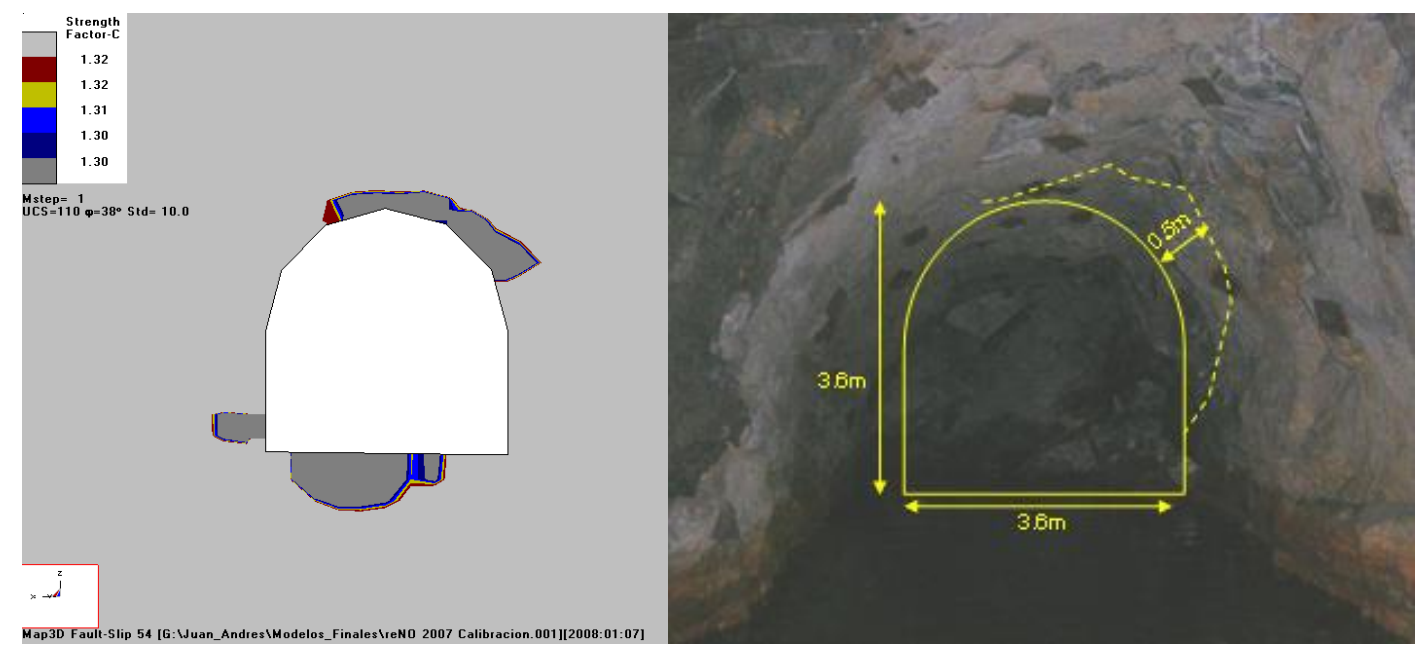

Figure 9 Example of one of the validation exercises done to estimate drift overbreak

\section{Conclusions and recommendations}

The results obtained from the different validation exercises for the Map3D model lead to the conclusion that the model can make predictions with an acceptable error for the prefeasibility stage of the NML project. For the evaluation of the stress state in specific zones, the model shows a mean error of $40 \%$ in the stress tensor. This error percentage is below the one obtained by similar mine-scale models and somewhat higher than the one obtained by the other mine-scale models made for the Project (mine-scale model, Itasca (2007) with 37\% error). On the other hand, when validating against field damage, such as drift overbreak and rock mass deterioration due to abutment stress, it is observed that the error obtained in the model is $20 \%$, something that allows predicting the behaviour of the rock mass due to the mining activity with an error level that is quite low for the scale and simplicity of the model.

The error obtained allows reliable predictions both at mine-scale and at drift-scale, making it possible to evaluate mining front geometries, mining macro sequences, and large caves and drifts stability.

The pre-mining stress state is the most important input parameter for the model. For this study, it was considered that the pre-mining stress state is given by the east-west compressive condition generated by the Nazca plate subduction under the South American plate.

Although, for the modelling process, only the elastic properties of the CMET and Braden Breccia lithologies were used, for the safety factor calculation, the rock properties corresponding to the sector under study were actually used.

For future works, it is recommended to include lithologies, from which Young's modulus differs in at least $30 \%$ from the CMET, because lower values do not generate important changes in the stress magnitude. In addition, as a future action, major structures will be included in the model and more details will be studied about the pre-mining stress orientation.

\section{Acknowledgements}

The authors want to thank Codelco Chile VCP for the permission to publish this study, based on a document prepared for the New Mine Level Project Geomechanics Area (Vasquez et al 2008a). Also we want to express our gratitude to Mr Patricio Cavieres for his contribution in this study.

\section{References}

Celhay, F., Pereira, J. and Burgos, L. (2005) Geology and Resources for the New Mine Level, Pre-feasibility Engineering stage, SGL-I-030/05. Internal report Codelco-Chile.

Hoek, E., Carranza-Torres, B. and Corkum, B. (2006) El Criterio de rotura de Hoek-Brown, Edición 2002. 
Itasca S.A. (1998) Updating of Mine-Scale 3D Numerical Models. Prepared for and funded by the Engineering and Planning, MINCO, Codelco Chile - El Teniente Division.

Itasca S.A. (2007) 3D Stress Model and Mining Macro Sequences for the New Mine Level Project, Codelco Chile - El Teniente Division, Study NNM-E-02. Prepared for and funded by the New Mine Level Project, VCP.

Karzulovic, A., Cavieres, P. and Gonzales, G. (2006) El Teniente Mine Conceptual Field Stress Model, Study DT-CG2006. Prepared for and funded by the New Mine Level Project, VCP.

Stress Map: web site www.wsm.physik.uni.karlsruhe.de

Vásquez, P., Cavieres, P. and Jarufe, J. (2008a) Report NNM-ICO-GEM-INF-008, 3D Stress Model for the New Mine Level Project (Map3D).

Vásquez, P., Cavieres, P., Jarufe, J., Bahamondes, C. and Valdivia, R. (2008b) Report NNM-ICO-GEM-INF-010, Geomechanical recommendation for the size, shape and decoupling of caving front. New Mine Level Project.

Wiles, T. (2007) Map3D Manual.

Wiles, T. (2006) Map3D Course Notes, @ Mine Modelling Pty Ltd 2006. 